\title{
Automatic Classification of the Movements of Directed and Undirected Subviral Particles
}

\begin{abstract}
The development of drugs against pathogens that cause hemorrhagic fever, such as Marburg and Ebola virus, requires researchers to gather much information about the virus. The accelerating of the research process is of great interest; therefore a new algorithm was developed to analyze intracellular processes. The algorithm will classify the motion characteristics of subviral particles in fluorescence microscopic image sequences of Ebola or Marburg virusinfected cells. The classification is based on the calculation of mean squared displacement. The results look promising to distinguish different particle tracks in active and passive transport. The paper ends with a discussion.
\end{abstract}

Keywords: subviral particles mean squared displacement, fluorescence microscopy, movement patterns.

https://doi.org/10.1515/cdbme-2020-3038

\section{Introduction}

The current pandemic outbreaks show how important the rapid developments of effective medicines are. A recent example is the coronavirus (COVID-19) pandemic [1]. The research demands a high amount of information to be collected.

Over the last few years, the Ebola virus has been a big issue. The reason for this is its high mortality rate of almost $50 \%$ [2]. In case of Ebola or Marburg viruses, an accurate knowledge about the motion characteristics of subviral particles in infected cells helps to understand the effects of potenital medicines on the pathogens. Therefore, scientists of the Institute for Virology of the Philipps-University Marburg have developed a method to make subviral particles in fluorescence live-cell images visible [3].

\footnotetext{
${ }^{*}$ Corresponding author: Michelle Kaak: Institut für Biomedizinische Technik (IBMT), FB Life Science Engineering (LSE), Technische Hochschule Mittelhessen (THM) - University of Applied Sciences, Gießen, Germany, e-mail:

michelle.kaak@Ise.thm.de

Andreas Rausch, Thomas Schanze: Institut für Biomedizinische Technik (IBMT), FB Life Science Engineering (LSE), Technische Hochschule Mittelhessen (THM) - University of Applied Sciences, Gießen, Germany
}

Manual analysis of the subviral particles is too timeconsuming and could lead to non-reproducible results [4]. For that reason, algorithms have been developed to detect and track the particles automatically. The Algorithms were presented by Kienzle et al. [5] and by Rausch et al. [6] in previous works. They operate on fluorescence microscopic image sequences that were provided by the workgroup of Prof. Dr. Stefan Becker, Institute for Virology, Marburg. Flat cells are used, so the third dimension can be neglected and the algorithms can provide two-dimensional coordinates for the tracked particles. The image of an infected cell with the tracking results provided by the algorithm by Rausch et al. [6] is shown in Figure 1.

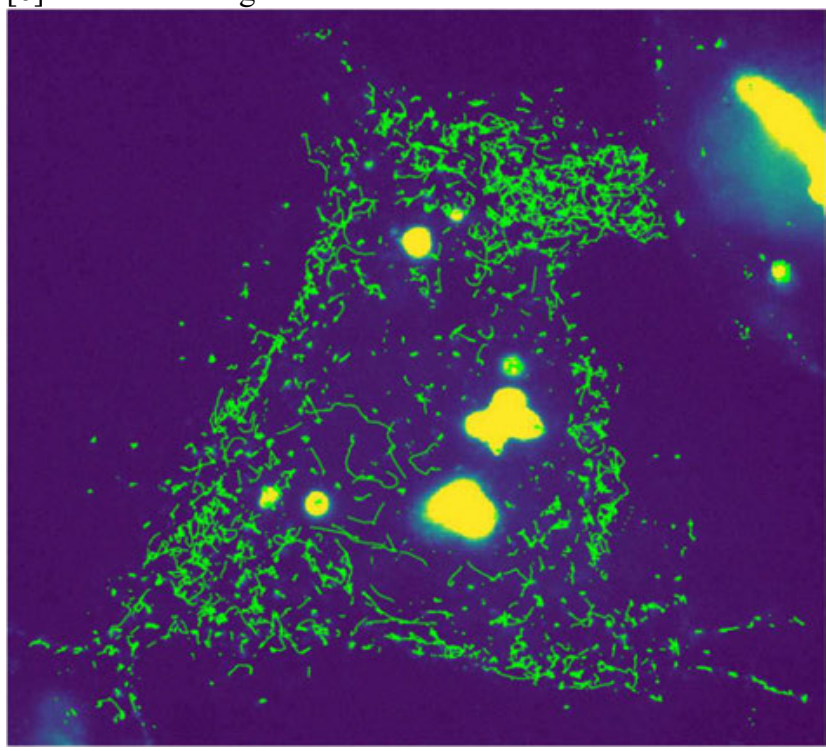

Figure 1: Real Ebola-virus infected cell recorded with a fluorescence microscope. The green lines show the detected particle tracks. The yellow areas indicate various components in the cell.

\section{Methods}

The coordinates of the tracked subviral particles are the basis for the presented method. The particles' trajectories are formed by the coordinates. The goal is to classify the trajectory that is given by:

$$
\mathbf{r}(t)=[x(t), y(t)]^{\prime}
$$

where $x(t)$ and $y(t)$ are coordinates at time $t$. 


\subsection{Calculating the Mean Squared Displacement}

To classify objects, first the classes must be defined. The calculation of mean squared displacement (MSD) is a frequently used technique to distinguish molecular motion in biological processes. Thus, it is possible to differentiate directed from chaotic Brownian movements. [7-9]. The MSD $\left\langle r^{2}\right\rangle$ is the mean of the squared distances from the launching point to the locations of residence at varying times of a particle. It is determined by:

$$
\left\langle\mathbf{r}^{2}\right\rangle=1 / N \sum_{n=1}^{N}\left\|\mathbf{r}\left(t_{n}\right)-\mathbf{r}(0)\right\|_{2}^{2}
$$

where $N$ is the count of locations of the particle, $\mathbf{r}(0)$ is the initial point and $\mathbf{r}\left(t_{n}\right)$ is the position at different time points $t_{n}$.

The kind of movement can be derived from a regression of the calculated MSD values (see Figure 2). In this concept the movement patterns are classified by three models:

One of the movement types is normal diffusion (Brownian movement). This is characterized by a linear growth, which is modelled as follows:

$$
\left\langle r^{2}\right\rangle=2 n \cdot D \cdot t
$$

$n$ represents the dimension of the system - here it is 2 - and $D$ stands for the diffusion constant.

The second type is anomalous diffusion, where an obstacle hinders the diffusion. It is denoted by a decreasing slope in the MSD curve. The path follows the power law:

$$
\left\langle r^{2}\right\rangle=4 \cdot D \cdot t^{\alpha}
$$

where $\alpha<1$ is the factor for the anomaly of the movement and $2 n$ is substituted by 4 , since $n$ is equal to 2 .

The combination of directed movement with diffusion share (diffusion with drift) results from active transport and is modelled as:

$$
\left\langle r^{2}\right\rangle=4 \cdot D \cdot t+v^{2} \cdot t^{2}
$$

where $v$ is the speed of the directional movement.

The introduced algorithm evaluates the MSD and fits the corresponding functions to the resulting curves by using the nonlinear least squares estimator. To save computation time, Equation (3) has been excluded from the algorithm because it can be substituted by (4) if $\alpha=1$ and also by (5) if $v^{2} \cdot t^{2}=0$. By minimizing the number of equations, a separation into three classes is much faster and more effective. The algorithm was coded in Python 3.7.

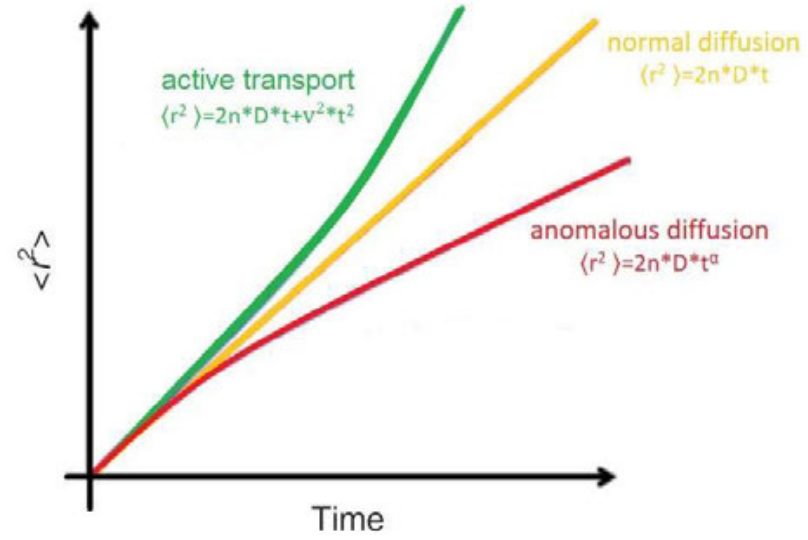

Figure 2: Mean squared displacement $\left\langle r^{2}\right\rangle$ depending on time for different movement types. The green curve stands for the active transport and is described by Equation (5). The orange plot increases linearly, which indicates diffusion. The corresponding Equation is (3). Abnormal diffusion is defined by Equation (4).

\subsection{Classification}

To identify the kind of motion, the fits are compared to each other and the more appropriate one is chosen. The fits are evaluated by using the mean square error (MSE).

$$
\operatorname{MSE}(Y, \widehat{Y})=1 / n \sum_{i=1}^{n}\left(Y_{i}-\widehat{Y}_{i}\right)^{2}
$$

where $Y_{i}$ are the actual values and $\hat{Y}_{i}$ are the approximate values. The closer the MSE is to zero, the better the fit.

In order to compare and normalize the results for the different functions, a contrast function is used:

$$
C=\frac{M S E 1-M S E 2}{M S E 1+M S E 2}
$$

where MSE1 is the mean squared error for Equation (5) and MSE2 is the mean squared error for Equation (4). The resulting value is between -1 and 1 , based on the type of motion. A value around -1 is a sign of an active transport, while a value close to 1 points to an anomalous diffusion. If the value is nearly zero, both equations fit equally well, which represents normal diffusion. The limits between the three types of motion are $\pm 1 / 3$. The values for all subviral particles can be illustrated by a distribution function. This allows a fast insight into the frequency of the movement types. The particle tracks are labeled in color for their movement type. A green color is applied to the particles with directional motion. Undirected movement is marked yellow for normal diffusion and red for anomalous diffusion. 


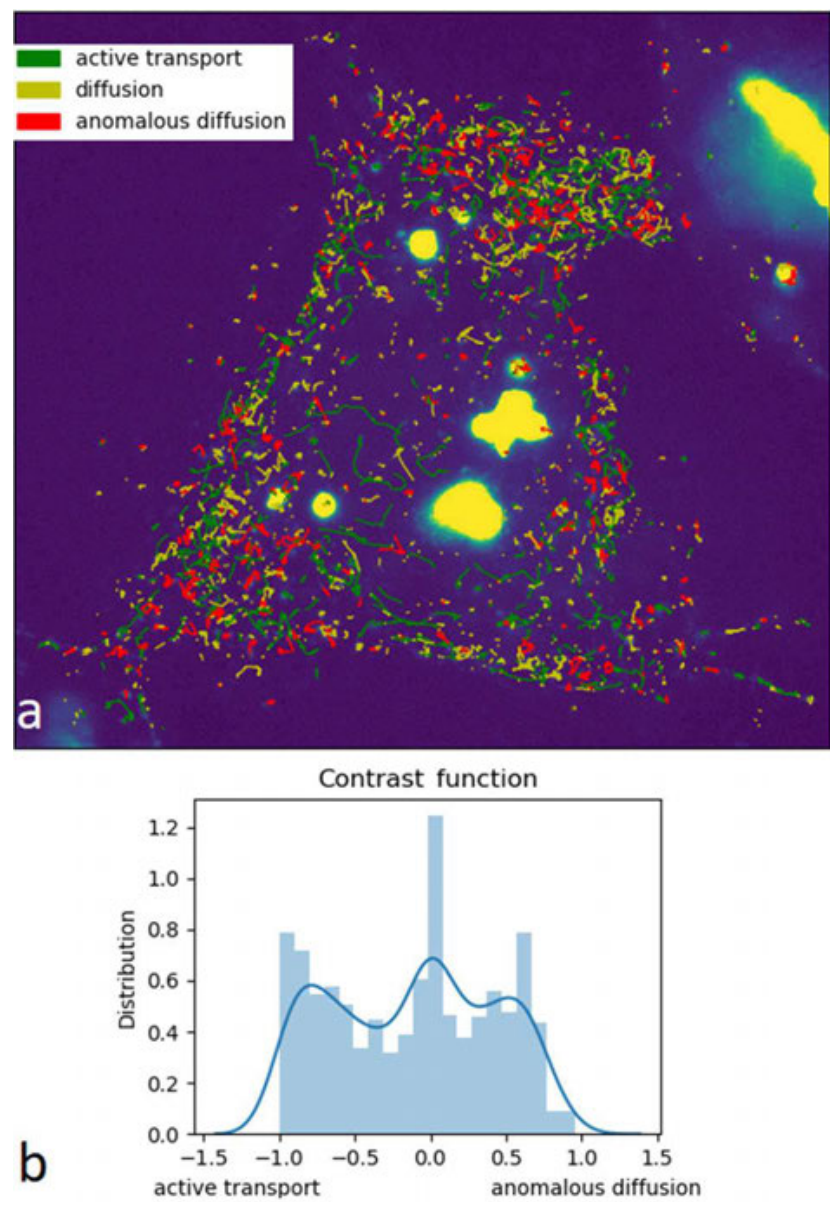

Figure 3: a) Classified tracks: The same infected cell as in Figure 1. The color-coded tracks illustrate the different types of movement and are explained in the legend. b) Representation of the distribution of the different types of movement. The trimodal distribution function illustrates the three types of movement that are present in the cell.

\section{Results}

The newly developed algorithm classifies the movement of subviral particles and assigns them a color in accordance with their class for visualization. The colored tracks are superimposed on the image of the cell for a visual result. By means of the colour of the track, it can be immediately recognised with which type of movement the particles are moving. Additionally, a frequency distribution is plotted in combination with a multi-Gaussian kernel density estimate. The trimodal course shows the distribution between the prevalent movement types. An example is shown in Figure 3.

For a more detailed visualization, individual particles can be displayed with their MSD curve. The curve is plotted with the two possible fits and is highlighted in the color of the most suitable one. Figure 4 shows the result for an exemplary particle that is actively moving. The course of the MSD describes the same direction as the fit created with Equation (5). The same applies to the subviral particle that moves with anomalous diffusion but it follows Equation (4). This can be seen in Figure 5. If the situation occurs that alpha tends towards 1 and $v$ (particle velocity) towards 0 , the course of both curves is the same. This is always the case when a diffusion movement is involved. The projection of a particle moving in this pattern is depicted in Figure 6.

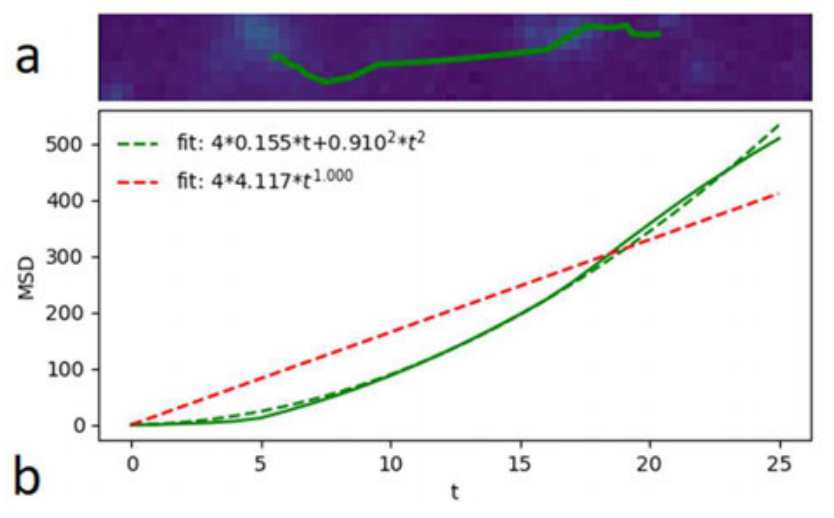

Figure 4: a) Selected track of a particle that moves in a directed manner. b) Plot of the MSD curve (solid green line) of the particle shown in a) with the possible fits that were adjusted according to Equation (4) (dashed red line) and Equation (5) (dashed green line). Due to the better fit of Equation (5) the MSD curve was colored green.

\section{Discussion}

In this paper, a new algorithm was presented that is intended to accelerate and simplify the analysis of subviral particles. The basis is the calculation of the MSD which is frequently used to classify motion of cells [7-9]. The course of the MSD curve varies depending on the type of movement. In the process of automatically classifying these curves, estimators are adapted to the data points. According to the best fit, the particle tracks are displayed in color, thus enabling quick identification of the type of motion. The color-coded tracks can be used to locate hotspots of a particular type of motion in the cell. The difference between active and passive motion could show whether a particle is untreated or treated and therefore still healthy or unhealthy. The frequency distributions can be particularly revealing when drugs are added. If a medication is working and the active transport of the subviral particles is expected to be suppressed, this can be immediately seen from the change of the frequency distribution. 


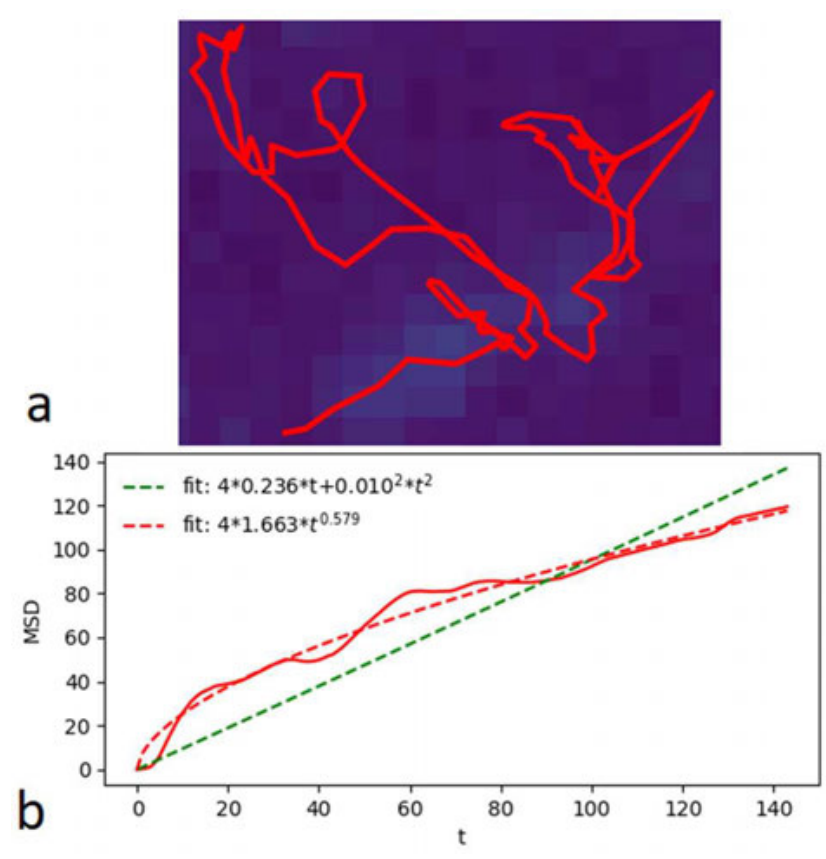

Figure 5: a) Close-up of a particle whose shape suggests anomalous diffusion. b) Plot of the MSD curve (solid red line) of the particle shown in a) with the possible fits.

Examining the results, it is noticeable that some particles change their mode of motion. The division into different phases could be useful to determine the estimated time and location at which the directional particles change their motion type and only move by diffusion. In further work, methods to divide motion patterns into phases will be investigated. Additionally, the classified traces will be validated by experts. For this purpose, the traces are classified by experts and the results are compared with those of the algorithm to verify our approach.

\section{Author Statement}

Research funding: The author state no funding involved. Conflict of interest: Authors state no conflict of interest. Informed consent: Informed consent has been obtained from all individuals included in this study. Ethical approval: The research related to human use complies with all the relevant national regulations, institutional policies and was performed in accordance with the tenets of the Helsinki Declaration, and has been approved by the authors' institutional review board or equivalent committee.

\section{Acknowledgment}

We thank the whole team of the Institute for Virology, Philipps-Universität Marburg, especially Stephan Becker and Olga Dolnik for ongoing collaboration and, in particular, for

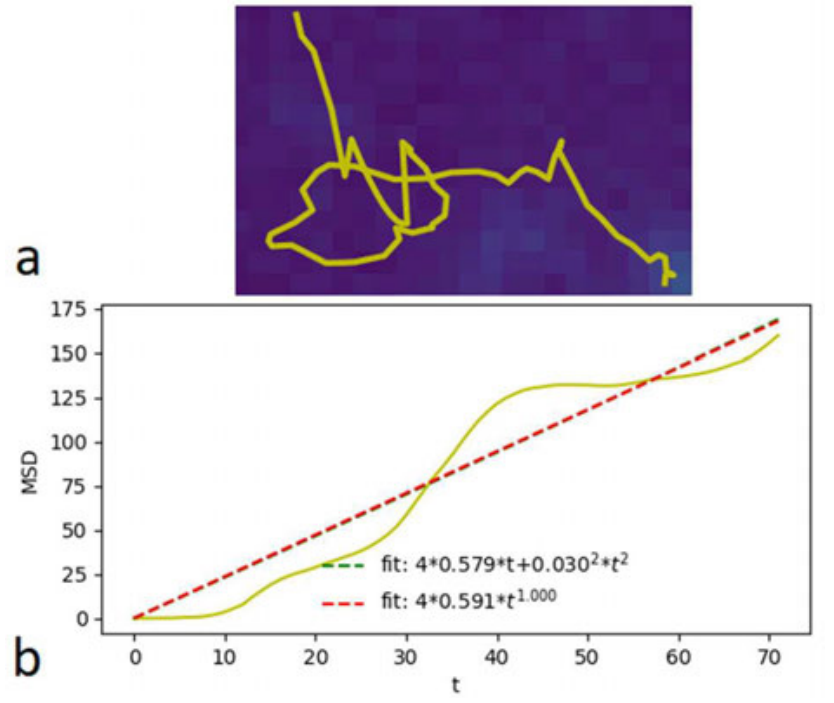

Figure 6: a) Close-up of a particle whose shape suggests diffusion. b) Plot of the MSD curve (solid yellow line) of the particle shown in a. The two fits have the same course.

providing the fluoroscopic image sequences in order to develop and test our methods.

\section{References}

[1] "Coronavirus disease 2019 (COVID-19)" (Situation Report 51). World Health Organization (WHO). 11 March 2019.

[2] "Ebola virus disease" (Press release). World Health Organization (WHO). 12 February 2018.

[3] Schudt G, Kolesnikova L, Dolnik O, Sodeik B, Becker S. Live-cell imaging of Marburg virus-infected cells uncovers actin-dependent transport of nucleocapsids over long distances. Proc Natl Acad Sci. 2013;110(35):14402-7.

[4] Müller D, Rausch A, Dolnik O, Schanze T. Comparing human and algorithmic tracking of subviral particles in fluorescence microscopic image sequences. Current Directions in Biomedical Engineering. Vol. 3. 2017.

[5] Kienzle C, Schudt G, Becker S, Schanze T. Multiple subviral particle in fluorescence microscopy sequences. Bildverarbeitung für die Medizin. Springer-Verlag. 330-335. 2014.

[6] Rausch A, Müller D, Schanze T. Improvement of a subviral particle tracker by the use of a LAP-Kalman-algorithm. Current Directions in Biomedical Engineering. Vol. 2. 2016.

[7] Saxton M.J, Jacobson K, Single-particle tracking: applications to membrane dynamics. Annu Rev Biophys Biomol Struct 26: 373-399. 1997.

[8] Ruthardt N, Lamb D.C, Bräuchle C, Single-particle tracking as a Quantitative Microscopy-based Approach to Unravel Cell Entry Mechanisms of Viruses and Pharmaceutical Nanoparticles. Molecular Therapy vol. 19 no. 7, S.11991211. 2011.

[9] Qian H, Sheetz M.P, Elson E.L, Single particle tracking. Analysis of diffusion and flow in two-dimensional systems. Biophys. J 60: 910-921. 1991. 